\title{
INFLUENCIA DO ÍNDICE DE MASSA CORPORAL NA FORÇA MUSCULAR DE MEMBRO INFERIOR EM IDOSAS PARTICIPANTES DA UNIVERSIDADE ABERTA DA TERCEIRA IDADE
}

Elaine Aparecida Lozano da Silva ${ }^{1}$, Lucas Oliveira Klebis ${ }^{1}$, Ana Caroline Rippi Moreno ${ }^{1}$, Maria Carolina Rodrigues Salini ${ }^{2}$, José Henrique Piedade Cardoso ${ }^{1}$, Regina Celi Trindade Camargo ${ }^{3}$.

Universidade Estadual Paulista - UNESP, ${ }^{1}$ Pós-Graduação em Fisioterapia, ${ }^{2}$ Graduação em Fisioterapia, ${ }^{3}$ Departamento de Fisioterapia, Presidente Prudente, São Paulo. E-mail: elaine lozano@hotmail.com

\section{RESUMO}

No decorrer do envelhecimento ocorrem modificações na força muscular e podem sofrer influências negativas por meio do índice de massa corporal. Trata-se de um estudo transversal com idosas ativas. Foi realizada a coleta da idade, peso, altura e aplicação do teste Sentar e Levantar. As participantes foram subdividas em 3 grupos de acordo com o IMC: Abaixo do peso (G. Abaixo), Ideal (G. Normal) e Acima do peso (G. Acima). $O$ estudo foi composto por 52 idosas, com idade média 71,0 $\pm 7,6$ anos e IMC 27,7 $\pm 4,4$ $\mathrm{Kg}$. Idosas do grupo $\mathrm{G}$. Abaixo apresentaram média de $15 \pm 2,9$ repetições, $\mathrm{G}$. Normal 13,6 $\pm 2,7$ repetições e G. Acima 12,7 $\pm 2,3$ repetições. Pode-se concluir que o melhor desempenho de força ocorre em valores de IMC normal e baixo, podendo o peso excessivo influenciar negativamente na força muscular de membros inferiores.

Palavras-chave: envelhecimento, força muscular, obesidade, composição corporal, exercício.

\section{INFLUENCE OF BODY MASS INDEX IN STRENGTH OF LOWER LIMB MUSCLES IN OLDER WOMEN PARTICIPANTS OF OPEN UNIVERSITY OF THIRD AGE}

\begin{abstract}
In the course of aging changes in muscle strength occurs and may suffer negative influences of body mass index. A cross-sectional study was conducted with active older women. It was collected age, weight, height and applied the Sitting-Rising test. The participants were subdivided into three groups according to BMI: Underweight (G. under), Ideal (G. normal), Overweight (G. over). The study was composed by 52 elderly women, with $71.0 \pm 7.6$ years and BMI $27.7 \pm 4.4 \mathrm{~kg}$. The women at G.under group had a mean of $15 \pm 2.9$ repetitions, G. Normal $13.6 \pm 2.7$ repetitions and G. over $12.7 \pm 2.3$ repetitions. It can concludes that the best strength performance occurs in BMI below and ideal, witch means that the overweight can negatively influence muscle strength of lower limbs.
\end{abstract}

Keywords: aging, muscle strength, obesity, body composition, exercise. 


\section{INTRODUÇÃO}

Atualmente acontece um aumento gradativo de idosos no Brasil, o que ocorre diante do avanço das áreas da saúde, que agem por meio de medidas preventivas, objetivando a melhoria da saúde e prevenção do desenvolvimento de doenças crônico-degenerativas na população idosa1. Nas últimas décadas, ocorreu aumento da população com idade igual ou superior a 65 anos, sendo que estes ocupavam apenas $4,8 \%$ da população brasileira em 1991, seguido de 5,9\% em 2000 e 7,4\% em 2010, segundo o Instituto Brasileiro de Geografia e Estatística [IBGE]2.

No período de envelhecimento, ocorrem modificações fisiológicas no sistema musculoesquelético, que podem levar a uma redução de massa corporal e força muscular, além de aumento do tecido adiposo. A força muscular começa a apresentar modificações importantes após os 50 anos de idade, podendo decrescer entre $8 \%$ e $15 \%$ por década, resultando em uma redução gradativa com o passar dos anos, que se acentua após a sexta década de vida3.

Além disso, o idoso pode apresentar redução da massa muscular que, quando acentuada, pode estar associada à patologia denominada sarcopenia, comprometendo a força muscular e a funcionalidade do indivíduo4.

Associadas à sarcopenia estão as alterações do equilíbrio e da massa corporal, que podem desenvolver a Síndrome da Fragilidade, aumentando os risco de quedas, fraturas, hospitalização e dependências na funcionalidade do idoso5.

Além disso, por meio da verificação do estado nutricional pelo Índice de Massa Corporal (IMC = $\mathrm{kg} / \mathrm{m} 2$ ) observa-se que a má nutrição (baixa massa corporal e/ou obesidade) é comumente encontrada nos indivíduos idosos, sendo que cerca de $1 / 3$ da população adulta e idosa apresentam tendência crescente para o excesso de peso nas últimas décadas6. A obesidade é classificada pelo excesso de tecido adiposo no organismo e pode estar associada a situações patológicas, tais como: doenças cardiovasculares, osteomusculares e neoplásicas, contribuindo também para o desenvolvimento de fragilidade e da mortalidade de idosos7.

As alterações na distribuição da gordura corporal e a obesidade têm sido relacionados à redução da capacidade funcional, podendo a obesidade estar associada a dificuldades na realização de determinadas tarefas que necessitam de força e flexibilidade muscular 8,9. Assim sendo, o objetivo do presente estudo é avaliar a influência do IMC em idosas ativas com a força muscular de membros inferiores (MMII) utilizando o teste Sentar e Levantar.

As alterações na distribuição da gordura corporal, o baixo peso e a obesidade têm sido relacionados à redução da capacidade funcional, podendo essa estar associada a dificuldades na realização de determinadas tarefas que necessitam de força e flexibilidade muscular ${ }^{8,9}$. Assim sendo, o objetivo do presente estudo é comparar valores de força muscular de membros inferiores em idosas segundo categorias de IMC.

\section{METODOLOGIA}

Foi realizado um estudo transversal com idosas participantes do programa de fisioterapia preventiva em geriatria e gerontologia da Universidade Aberta à Terceira Idade (UNATI), vinculado ao Setor de Geriatria e Gerontologia do departamento de Fisioterapia da FCT/UNESP. Este conta com um protocolo de exercícios físicos, supervisionado por Fisioterapeutas, composto de alongamentos, exercícios para fortalecimento muscular, flexibilidade, coordenação, equilíbrio, memória e relaxamento. 0 programa conta com três sessões semanais, com uma hora de duração.

O estudo foi aprovado pelo Comitê de Ética, CAAE N ${ }^{\circ} 56496816.3 .0000 .5402$. Foi realizada a coleta de dados para caracterização das participantes, sendo: idade, peso e altura para cálculo do IMC. As participantes foram subdividas em 3 grupos, de acordo com seu resultado final do IMC: Abaixo do peso (G. Abaixo), quando resultado menor a $22 \mathrm{~kg} / \mathrm{m}^{2}$; Ideal (G. Normal) quando maior ou igual a $22 \mathrm{~kg} / \mathrm{m}^{2}$ e menor ou igual a $27 \mathrm{~kg} / \mathrm{m}^{2}$; Acima do peso (G. Acima), quando maior a $27 \mathrm{~kg} / \mathrm{m}^{2}{ }^{10}$.

Após a subdivisão, foi realizado a análise da força muscular de MMII por meio do teste "sentar e levantar", onde foi solicitado para a voluntária que se sentasse e levantasse de uma cadeira, encostada na parede, cinco vezes seguidas, a fim de verificar a segurança da realização da tarefa. Após isso, era solicitado que se iniciasse o teste na posição sentada sobre a cadeira, com os braços cruzados sobre o tórax, e se levantasse e sentasse novamente o maior número de vezes e o mais rápido possível, sendo cronometrado 
o tempo de 30 segundos. O teste era considerado concluído com êxito quando não ocorriam intercorrências, como pausas, mal-estar, e quaisquer alterações que gerassem desconforto à participante.

Para análise estatística foi utilizado o software PRISMA, apresentando média, desvio padrão e valor de $p$ segundo o teste ANOVA / One Way, utilizando como teste post-hoc Turkey. Foi considerado diferença significante quando $p<0,05$.

\section{RESULTADOS}

O estudo contou com uma amostra de 52 participantes, com média de idade $71,0 \pm 7,6$ anos e IMC $27,7 \pm 4,4$ quilos

Após a análise geral, as participantes foram subdividas em 3 grupos de acordo com o resultado final do IMC por meio da classificação sugerida por CERVI A et al. (2005). Na tabela I observa-se a caracterização de cada grupo, com número de participantes, media e desvio padrão da idade, peso, altura e IMC.

Tabela I. Subdivisão dos participantes com, média, desvio padrão e valor de $\mathrm{p}$ calculado com análise estatística One Way.

\begin{tabular}{l|cccc}
\hline & G. Abaixo & G. Normal & G. Acima & $p(<0,05)$ \\
& & & & \\
\hline Número (N) & 4 & 19 & 28 & - \\
Idade & $71 \pm 5,5$ & $71,6 \pm 8,0$ & $70,7 \pm 7,7$ & 0,9244 \\
Peso & $44,4 \pm 4,3$ & $57,6 \pm 7,4$ & $74,6 \pm 8,1$ & $0.0001^{*}$ \\
Altura & $1,51 \pm 0,02$ & $1,53 \pm 0,06$ & $1,54 \pm 0,05$ & 0,5018 \\
IMC & $19,3 \pm 1,2$ & $24,4 \pm 1,4$ & $31,1 \pm 2,1$ & $0.0001^{*}$ \\
\hline
\end{tabular}

*significante vs G. Abaixo, G. Normal e G. Acima ( $p<0,001)$.

Na figura I, está presente a análise estatística, comparando os resultados do teste sentar e levantar dos 3 grupos. Apesar da ausência de diferença estatística significante $(p=0,1983)$, é possível visualizar diferença entre os grupos. Observa-se que as participantes do G. Abaixo apresentaram média de $15 \pm 2,9$ repetições, G. Normal média de 13,6 $\pm 2,7$ repetições e G. Acima 12,7 $\pm 2,3$ repetições.

\section{Teste sentar e levantar}

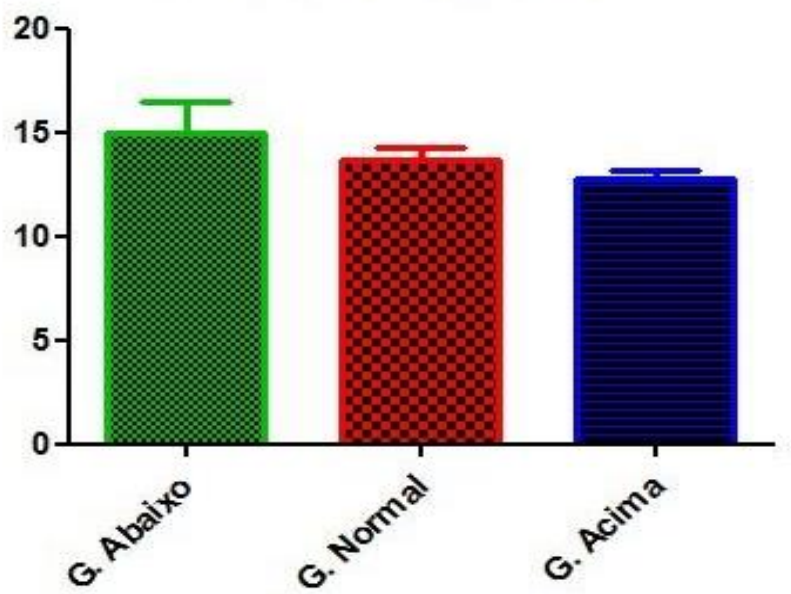

Figura I. Correlação do teste sentar e levantar com o IMC dos participantes. 
Em valores percentuais, observa-se uma redução de $10 \%$ ao comparar o G. Normal com o G. Abaixo, e de $15 \%$ ao comparar o grupo G. Acima.

\section{DISCUSSÃO}

Diante dos dados expostos, foi possível observar média de idade de 71,0 $\pm 7,6$ anos para as idosas. Mesmo após a subdivisão de 3 grupos de acordo com seu IMC, a média permaneceu acima de 70 anos.

Entende-se que a categorização funcional do idoso não depende apenas da idade, mas também de sexo, estilo de vida, saúde, fatores sócio-econômicos e influências constitucionais, estando provado, assim, que não há homogeneidade na população idosa ${ }^{11}$. $\mathrm{O}$ avanço da idade está muitas vezes associado com uma evolução gradual das características do envelhecimento, existindo envelhecimento primário, secundário e terciário ${ }^{12}$.

O envelhecimento primário é geneticamente determinado ou pré-programado, sendo presente em todas as pessoas (universal) ${ }^{13}$. 0 indivíduo nesse estágio está sujeito à concorrente influência de vários fatores determinantes para o envelhecimento, como exercícios, dieta e estilo de vida.

Diante dos resultados expostos no presente estudo, observa-se que nenhum dos três grupos apresentou escore inferior ao ideal no que se refere ao teste sentar e levantar, indicando que todas as participantes do mesmo apresentavam bons índices de força muscular de MMII. Esse fato pode estar relacionado com a prática regular de atividade física, que atua de forma benéfica ao idoso, podendo prevenir alterações primárias ao envelhecimento.

Foi possível observar ainda que, embora não exista diferença estatística entre os resultados, o menor resultado obtido no teste sentar e levantar foi referente ao grupo G. Acima, sendo $15 \%$ menor que os do grupo G. Abaixo. Um trabalho desenvolvido por Teixeira et al. (2014) apresentou a relação da influência do IMC com a redução de força muscular ${ }^{14}$. Esse fato corrobora com os dados apresentados no presente estudo, sendo que mesmo as participantes do G. Acima obtendo resultados dentro do escore satisfatório, sugere-se que quanto menor o IMC, melhor o desempenho no Teste sentar e levantar, desse modo, maior a força muscular de membros inferiores.

Em estudo realizado com 54 idosas participantes do Programa de Atividade Física da Terceira Idade do Centro de Desportos (CDS) da Universidade Federal de Santa Catarina (UFSC), foram apresentados valores positivos para força muscular de membros inferiores quando realizada reavaliação, partindo da média de 14,2 para 17,3 repetições ${ }^{15}$. Assim se faz necessário que o presente estudo tenha continuidade, com uma futura reavaliação das participantes e verificação dos efeitos da prática de exercício físico no aumento ou manutenção da força muscular nos membros inferiores nessa população.

O equilíbrio corporal, bem como a força muscular de membro inferior, está intimamente ligados ao medo que os idosos têm de cair ${ }^{16}$. Sendo assim, com valores de IMC dentro da média, junto com pratica de exercício físico regular, o idoso mantém-se dentro de um escore satisfatório no teste sentar e levantar, este diretamente relacionado com a força muscular de membros inferiores, e desse modo apresentando menor risco de quedas.

Entretanto o excesso de peso está também ligado ao desenvolvimento de osteoartrite no joelho, fato esse que pode afetar o desempenho motor do idoso, em decorrência da limitação dos movimentos e sobrecarga na articulação, podendo assim afetar negativamente o desempenho do teste sentar e levantar ${ }^{17}$.

Dessa forma, pode se concluir que o desempenho de força muscular dos membros inferiores pode ser influenciado pelos valores de IMC, mas ainda são necessários estudos para comprovar quantitativamente tal achado.

\section{CONFLITO DE INTERESSES}


Os autores declaram não haver qualquer potencial conflito de interesses que possa interferir na imparcialidade deste trabalho científico.

\section{REFERÊNCIAS}

1. QUEIROZ, D.B.; ARAÚJO, C.M.; NOVAIS, M.M.; LORENA, C.O.; ANDRADE, L.A.A.; REIS, L.A. Funcionalidade, aptidão motora e condições de saúde em idosos longevos residentes em domicílio. Arq. Ciênc. Saúde. 2016; 23(2) 47-53. DOI: http://dx.doi.org/10.17696/2318$\underline{3691.23 .2 .2016 .281}$

2. Instituto Brasileiro de Geografia e Estatística (2010). Censo demográfico 2010: resultados preliminares do universo. Recuperado em setembro, 2012.

3. GARCIA, P.A.; DIAS, J.M.D.; DIAS, R.C.; SANTOS, P.; ZAMPA, C.C. Estudo da relação entre função muscular, mobilidade funcional e nível de atividade física em idosos comunitários. Rev Bras Fisioter, 2011; 10(1):15-22.DOI: http://dx.doi.org/10.1590/S1413-35552011000100005

4. MULLER, D.V.K.; TAVARES, G.M.S.T.; GOTTLIEB, M.G.V.; SCHNEIDER, R.H. Avaliação do equilíbrio corporal e da força isocinética de flexores e extensores de joelho de um idoso sarcopênico, diabético com deficiência visual total: estudo de caso comparativo. Rev. Aten. Saúde, 2016;14 (48) : 54-61. DOI: http://dx.doi.org/10.13037/ras.vol14n48.3616

5. VIEIRA, M.C.A.; SOUZA, C.F.; CÂMARA, S.M.A.; MATOS, G.S.R.; MOREIRA, M.A.; MACIEL, A.C.C. Relação entre força de preensão manual e força de membro inferior em mulheres de meia idade: um estudo transversal. Rev Bras Ativ Fis Saúde;2015; 20(5):467-475. DOI: http://dx.doi.org/10.12820/rbafs.v.20n5p467

6. SOUZA, R.; FRAGA, J.S.; GOTTSCHALL, C.B.A.; BUSNELLO, F.M.; RABITO, E.I. Avaliação antropométrica em idosos: estimativas de peso e altura e concordância entre classificações de IMC. Rev. Bras. Geriatr. Gerontol., 2013; 16(1):81-90. https://doi.org/10.1590/S1809$\underline{98232013000100009}$

7. OLIVEIRA, C.S.R. Contextualização da produção científica brasileira sobre obesidade em pessoas idosas. [Trabalho de Conclusão de Curso].Campina Grande: - Universidade Estadual da Paraíba, 2016.

8. APOVIAN, C.M.; FREY, C.M.; WOOD, G.C.; ROGERS, J.Z.; STILL, C.D.; JENSEN, G.L. Body masS index and physical function in older women. Obes Res. 2002;10(8):740-47. DOI: http://dx.doi.org/10.1038/oby.2002.101

9. FERRUCCI, L.; PENNIX, B.W.J.H.; LEVEILLE, S.G.; CORTI, M.C.; PAHOR, M.; WALLACE, R. Characteristics of nondisabled older persons who perform poorly in objective tests of lower extremity function. J Am Geriatr Soc. 2000; 48(9):1102-10. DOI: http://dx.doi.org/10.1111/j.1532$\underline{5415.2000 . t b 04787}$

10. CERVI, A.; FRANCESCHINI, S.C.C.; PRIORE, S.E. Análise critica do uso do índice de massa corporal para idosos. Ver. Nutri. 2005; 18 (6):765-775. DOI: http://dx.doi.org/10.1590/S1415$\underline{52732005000600007}$

11. SHEPHARD, R.J. Envelhecimento, atividade física e saúde. São Paulo: Phorte; 2003. 
12. FECHINE, B.R.A.; TROMPIERI, N.O processo de envelhecimento: as principais alterações que acontecem com o idoso com o passar dos anos. 2012;1(20). DOI: http://dx.doi.org/10.6020

13. NETTO, M.P. História da velhice no século XX: Histórico, definição do campo e temas básicos. In E.V. Freitas., L. Py., A.L. Néri., F.A.X. Cançado., M.L. Gorzoni, M.L e S.M. Rocha (Eds.), Tratado de Geriatria e Gerontologia. Rio de Janeiro: Guanabara Koogan, p.1-12, 2002.

14. TEIXEIRA, T.G.; TIBANA, R.A.; VIEIRA, D.C.L.; TAJRA, V.; BALSAMO, S.; ASANO, R.Y.; SILVA, A.O.; PRESTES, J. Comparação da força muscular entre mulheres de meia idade com e sem sobrepeso/obesidade. R. Bras. Ci e Mov. 2014; 22 (1): 11. https://doi.org/10.18511/01031716/rbcm.v22n1p5-11

15. GUIMARÃES, A.V.; LOPES, M.A.; FREITAS, C.L.R.; BENEDETTI, T.B. Estudo longitudinal da força em idosas praticantes de atividade física co $\mathrm{m} 70$ an os e mais. Estud. interdiscipl. envelhec., 2013; 18(1): $77-88$.

16. HAUSER, E.; SANDRESCHI, P.F.; PARIZZOTTO, D.; ARAÚJO, C.C.R.; MAZO, G.Z. Medo de cair e desempenho físico em idosos praticantes de Atividade física. Rev. Educ. 2015;26 (4):593-600. DOI: http://dx.doi.org/10.4025/reveducfis.v26i4.28442

17. BARBOSA, A.R.; SOUZA, J.M.P.; LEBRÃO, M.L.; MARUCCI, M.F.N. Estado nutricional e desempenho motor de idosos de São Paulo. Rev Assoc Med Bras 2007;53 (1):75-9. https://doi.org/10.1590/S0104-42302007000100024 